\title{
Neuroimaging Studies of Emotional Responses in PTSD
}

\author{
ISRAEL LIBERZON AND BRIAN MARTIS
}

Trauma Stress and Anxiety Research Group, VA Ann Arbor Healthcare System, Department of Psychiatry, University of Michigan, Ann Arbor,

Michigan 48109-0118, USA

\begin{abstract}
Neuroimaging research offers a powerful and noninvasive means to understand healthy as well as dysregulated emotional processing in healthy subjects and PTSD patients. Functional neuroimaging findings suggest specific roles for subregions of the medial prefrontal (mPFC), orbito frontal (OFC), anterior cingulate (ACC), and insular cortices as well as the sublenticular extended amygdala (SLEA) and hippocampus in various components of emotional processing. Some of the same regions appear to be associated with emotional response to trauma, and with symptom formation in PTSD. Neuroimaging findings of emotional processing in healthy subjects and PTSD patients are discussed, addressing the specific roles of cortical regions like $\mathrm{MPFC}, \mathrm{ACC}$, and insula, and their potential contribution to PTSD pathophysiology. Processes of cognitive-emotional interactions and social emotions are discussed in an attempt to synthesize the prefrontal findings in healthy subjects and PTSD patients. Further links between functional neuroanatomy of emotional responses and neuroendocrine stress regulation are proposed.
\end{abstract}

KEYWORDS: PTSD; emotion regulation; neural circuitry; functional neuroimaging; medial prefrontal cortex; anterior cingulated cortex; amygdala; hippocampus

\section{INTRODUCTION}

Over the past decade, both basic animal research and human neuroimaging studies have begun to outline specific neural circuitry dedicated to emotional functioning. ${ }^{1,2}$ This work has partially inspired hypotheses regarding dysfunction in this circuitry leading to the development and maintenance of posttraumatic stress disorder (PTSD). ${ }^{3-5}$ While these studies have provided useful initial information and have guided the beginning of functional neuroanatomical/neurophysiological research in PTSD, it is becoming increasingly clear that the scope of these studies does not fully capture the

Address for correspondence: Israel Liberzon, M.D., Trauma Stress and Anxiety Research Group, Department of Psychiatry, Ann Arbor VA Healthcare System, University of Michigan, 1500 E. Medical Center Drive UH9D 0118, Ann Arbor, MI 48109-0118. Voice: 734-764-9527; fax: 734-936-7868. e-mail: liberzon@umich.edu

Ann. N.Y. Acad. Sci. 1071: 87-109 (2006). (C) 2006 New York Academy of Sciences. doi: 10.1196/annals.1364.009 
complexity of changes occurring in trauma exposure and PTSD development. For instance, while the predominant notion of PTSD being a state of abnormal responsivity to threat is intuitively appealing and explains some aspects of PTSD (such as hypervigilence and hyperarousal) it does not address the mechanisms of such changes. It also does not explain important associated phenomena, such as intrusive thoughts and memories, generalization, avoidance and numbing; vulnerability and resilience factors; significant mood and substance related comorbidity; and chronicity of PTSD in some patients.

There is a growing understanding that additional mechanisms other than "hypersensitivity to threat" may be involved in PTSD pathophysiology, and that they need to be investigated and further integrated in conceptualization of PTSD. Some of these central neurobiological and psychological processes that appear very relevant to PTSD development, maintenance, and/or recovery include the phenomena of conditioning, habituation, stimulus generalization, and (resistance to) extinction; cognitive-emotional modulation (involving appraisal and reappraisal); and social and self-related emotional processing. Furthermore, to fully understand both healthy processes listed above, and the development of pathophysiology, the field needs to integrate functional neuroanatomical findings with those that come from neurochemistry, neurophysiology, and neuroendocrinology studies in PTSD. The aim of this focused article is to elaborate on some of these mechanisms as they may relate to the state of trauma exposure/PTSD and to initiate a discussion that may lead to more nuanced and integrative investigations in this area. The first part of the ensuing discussion briefly reviews what is currently known on the basis of functional neuroimaging studies of emotional responses in subjects with PTSD. These consist mainly of symptom provocation studies and symptom correlation, and cognitive activation studies as well as functional connectivity analyses. The second part of the discussion focuses on emotional processes and the implicated neural substrates in healthy subjects that require further study on account of their relevance to PTSD. These include neuroimaging studies (in healthy humans) of fear conditioning, habituation, and extinction; cognitive-emotional interactions; and self-related and social emotional processing. The third part of the review briefly discusses emerging literature that attempts to extend what is known of the functional neuroanatomy of central stress systems to the study of PTSD. Finally, potential future directions for PTSD research areas are proposed.

\section{Emotional Responses in PTSD Patients: Functional Neuroimaging Studies}

Functional neuroimaging studies in PTSD include symptom provocation paradigms and correlational analyses of brain activations and symptom severity, neurocognitive activation studies (probing the anterior cingulate cortex 
[ACC], amygdala, and hippocampal functioning), and functional connectivity analyses. The ensuing discussion is a selective review of such studies in PTSD.

\section{Symptom Provocation Studies}

Symptom provocation studies are the most abundant studies in PTSD involving multiple modalities including single-photon emission tomography (SPECT), positron emission tomography (PET), and functional magnetic resonance imaging (fMRI). They involve provoking symptoms while attempting to capture the underlying neural substrates (as gleaned from blood flow and blood oxygen level dependent [BOLD] effects) and employ trauma-related stimuli of an autobiographical nature (e.g., narrative scripts of personal trauma) or general nature (e.g., nonpersonalized pictures and sounds). Both types of stimuli reliably induce psychophysiological changes, such as elevated heart rate, skin conductance, and plasma catecholamines in PTSD subjects. ${ }^{6-10}$ These observations suggest that trauma exposure and/or the PTSD state is associated with neurobiological changes in stress-related systems including the hypothalamicpituitary-adrenal (HPA) axis and autonomic nervous system. These changes are understood to be coordinated by central neural mechanisms.

Rauch and colleagues ${ }^{11}$ first used individualized trauma scripts and $\left[{ }^{15} \mathrm{O}\right]$ $\mathrm{H}_{2} \mathrm{O}$ PET in a small and heterogeneous group of eight PTSD subjects and demonstrated increased regional cerebral blood flow (rCBF) in anterior paralimbic (right posterior medialorbito frontal cortex [OFC], insular, anterior temporal polar, and medial temporal cortex) and limbic structures (amygdala) in the provoked versus control contrast. The same group then used combat-related, emotionally negative, and neutral pictures paired with verbal descriptions (imagery) in a PET study to investigate the specificity of emotional processing in combat veterans with and without PTSD (seven each group), as well as to differentiate visual perception from visual imagery. Combat veterans with PTSD had increased rCBF in ventral ACC and right amygdala when generating mental images of combat-related pictures but had decreased $\mathrm{rCBF}$ in the ACC in the combat image viewing versus neutral image viewing contrast. ${ }^{11,12}$ Though these early studies had methodological limitations, such as small and heterogeneous sample size and the lack of adequate control groups that limited the generalization of their findings, they set the stage for more detailed studies into the neural substrate of the symptomatic PTSD state.

In an ensuing study we exposed three groups of subjects (14 combat PTSD subjects, 11 combat-exposed subjects without PTSD, and 11 combatunexposed healthy subjects) to combat sounds or white noise in two counterbalanced sessions and studied $\mathrm{rCBF}$ with ${ }^{99 \mathrm{~m}} \mathrm{Tc}$-hexamethylpropyleneamineoxime (HMPAO) SPECT. Only the PTSD group showed increased rCBF in the left amygdaloid region (for the main contrast of combat sounds-white noise). ${ }^{13}$ Another study using combat-related pictures and sounds and PET in 
10 combat veterans with and 10 without PTSD revealed decreased blood flow in medial prefrontal cortex (mPFC) (area 25) and other areas in response to traumatic pictures and sounds in PTSD patients, while non-PTSD control subjects activated the anterior cingulate (area 24) to a greater degree than PTSD patients. ${ }^{14}$

The same group also studied a different cohort of subjects (22 women with histories of childhood sexual abuse [CSA]; 10 of whom had PTSD) with exposure to traumatic and neutral scripts and PET. The PTSD group showed rCBF increases in posterior cingulate (area 31) and anterolateral prefrontal cortex (PFC) (superior and middle frontal gyri bilaterally, areas 9 and 10). The PTSD group also showed deactivation in the subcallosal gyrus region of anterior cingulate (area 25) with a failure of activation in an adjacent portion of anterior cingulate (area 32). ${ }^{15}$ In a similar cohort (16 subjects with CSA; 8 with PTSD), Shin and colleagues, using a script-driven imagery and PET study reported greater increases in $\mathrm{rCBF}$ in the OFC and temporal poles and deactivation of the medial prefrontal and left inferior frontal (Broca's) areas in the PTSD group versus the non-PTSD group in the traumatic versus neutral imagery contrast. ${ }^{16}$

Lanius and colleagues reported two studies where they used a script-driven symptom provocation paradigm and fMRI. The second study also included comparison of nontraumatic negative states - sad and anxious. They reported significantly decreased BOLD signal in the ACC (Brodmann's area 32) and the thalamus in the PTSD group to both the traumatic and nontraumatic emotional states conditions, suggesting that the earlier neuroimaging findings related to these areas in PTSD may not be specific to traumatic stimuli. ${ }^{17,18}$ Hendler and colleagues, reported findings from an fMRI study of combat veterans with and without PTSD where subjects viewed pictures with and without combat content in repeated versus novel presentations. Repeated presentations of the same combat visual stimuli resulted in less BOLD signal decrease in the lateral occipital cortex in PTSD subjects (vs. non-PTSD), suggestive of impaired habituation of the response to trauma-related stimuli. ${ }^{19}$

A more recent script-driven imagery and PET study of 17 Vietnam veterans with PTSD and 19 without PTSD showed rCBF decreases in the medial frontal gyrus for the traumatic versus neutral comparison in the PTSD group. This activity was inversely correlated with $\mathrm{rCBF}$ changes in the left amygdala and the right amygdala/periamygdaloid cortex. Only the male combat veteran subgroup (and not the female nurse veteran subgroup) showed increased $\mathrm{rCBF}$ in left amygdala. ${ }^{20}$

We recently conducted a $\left[{ }^{15} \mathrm{O}\right] \mathrm{H}_{2} \mathrm{O}$ PET, script-driven imagery study of emotionally evocative and neutral autobiographic events in 16 combat veterans with PTSD (PTSD patients [PP], 15 combat veterans without PTSD (combat controls [CC], and 14 healthy, age-matched, noncombat control subjects (noncombat controls $[\mathrm{NC}]$ giving us the ability to study changes that are trauma related (PP vs. NC and CC vs. NC) and PTSD specific (PTSD vs. CC). For the 
traumatic/stressful > neutral scripts, while all subjects deactivated the mPFC and activated the insula, the PP deactivated the rostral anterior cingulate cortex (rACC) more than both control groups (CC and $\mathrm{NC}$ ) that additionally showed ventromedial PFC (vmPFC) deactivation. Trauma exposure (i.e., PP and CC groups) was associated with decreased amygdala activity compared to NC. The findings observed only in the PTSD group (deactivation of the rACC) may reflect neural substrates specific to PTSD, whereas trauma-specific patterns (decreased amygdala activity) may represent potential compensatory changes to traumatic reminders. ${ }^{21}$

\section{Correlation With Cross-sectional Symptom Severity}

Several investigators have also used correlational analysis in neuroimaging data to attempt to understand the phenomenology of symptom genesis in PTSD (the correlation of imaging findings with measures of symptom severity). Osuch and colleagues correlated $\mathrm{rCBF}$ response with flashback intensity in a personalized, script-driven imagery PET paradigm in eight chronic PTSD subjects. ${ }^{22}$ rCBF correlated directly with flashback intensity in the brain stem, insula, and hippocampus, and inversely in the prefrontal, right fusiform, and medial temporal cortices. Similarly, in an fMRI study, Lanius and colleagues reported that 7 CSA subjects with PTSD and concomitant dissociative responses to symptom provocation by scripts had increased activation in the ACC, mPFC, and several other cortical areas compared to 10 control subjects. ${ }^{23}$ However, none of these activations correlated with either dissociative or flashback intensity. The small sample sizes and significant comorbidity of this cohort limit the interpretation of these findings. In a script-driven imagery and PET study reported above, ${ }^{20}$ Shin and colleagues found that in the PTSD group, for the traumatic condition, symptom severity [as measured by the total score on the Clinician-Administered PTSD Scale (CAP)] was positively related to $\mathrm{rCBF}$ in the right amygdala and negatively related to $\mathrm{rCBF}$ in medial frontal gyrus after controlling for depression severity score. In a recent block design fMRI study, investigators examined the time course of amygdala responses to trauma-relevant negative words, panic-relevant negative words (negative control condition), positive/safety words, and neutral words, in 9 predominantly sexual assault PTSD patients and 14 healthy controls. ${ }^{24}$ The PTSD group showed an increased left amygdala response to trauma-relevant negative versus neutral stimuli compared to controls in the first two (but not last two) runs, and this response correlated with the symptom severity (CAPS total score). Healthy controls showed the opposite pattern.

Thus, symptom provocation studies have implicated several anterior paralimbic and limbic structures in the symptomatic state of PTSD including the posterior medial OFC, the insula, and the medial temporal cortex. Several studies but not all have also demonstrated decreased or failure of activation in 
subregions of the mPFC and ACC. Increased responsivity of the amygdala has been observed in some studies, but has not been a consistent finding. Several issues of design and/or methodology may contribute to these divergent findings, including the nature of symptom provocation method (trauma imagery vs. external stimuli), experimental tasks (passive viewing vs. active recall), scanning methods, and relatively small sample sizes, all of which may effect the ability to activate and/or detect amygdala response. Several investigators have used correlational analyses to investigate the relationship between symptomprovoked activation and cross-sectional symptoms severity, but findings have been inconsistent for meaningful interpretation at this time.

\section{Cognitive Activation Studies}

Cognitive activation studies investigate implicated brain structures using a neurocognitive task that is expected to activate that region (a "probe"). Selectively activating a region without eliciting symptoms has a substantial advantage in that this overcomes the confound of the admixture of nonspecific anxiety responses. In one of the earliest of such studies, Semple and colleagues used $\left[{ }^{15} \mathrm{O}\right] \mathrm{H}_{2} \mathrm{O}$ PET and challenge with an auditory attentional task in 8 PTSD patients and 8 healthy controls with substance abuse and reported an $8-11 \%$ lower $\mathrm{rCBF}$ in the right angular gyrus during the attentional task. ${ }^{25}$ However, several methodological issues limited interpretation of this finding, including small sample size, substance abuse comorbidity, lack of trauma-exposed control subjects, and the potentially confounding contribution of baseline rCBF differences. Investigators have also used cognitive activation strategies to study implicated regions in PTSD, such as the amygdala, ACC, and hippocampus.

\section{Amygdala}

Using an innovative design, Rauch and colleagues compared amygdala responses in 9 PTSD subjects versus eight combat-exposed, non-PTSD subjects using a previously validated masked emotional faces paradigm. ${ }^{26}$ Fearful versus happy masked faces contrasts revealed exaggerated amygdala responses in the PTSD subjects. Moreover, the magnitude of these responses distinguished PTSD subjects with $75 \%$ sensitivity and $100 \%$ specificity. These findings are significant in that they provide evidence for increased amygdala responsivity to threat-related (but not necessarily trauma-related) stimuli in PTSD, as well as the ability of nonconscious threat-related stimuli to elicit such a response in PTSD.

\section{$A C C$}

Bremner and colleagues ${ }^{27}$ used the Stroop task (color Stroop, emotional Stroop, and control task) and $\left[{ }^{15} \mathrm{O}\right] \mathrm{H}_{2} \mathrm{O}$ PET to probe ACC function in 
12 women with early CSA-related PTSD and 9 abused women without PTSD. The PTSD group had a relative decrease in ACC blood flow during the emotional but not the color Stroop task, which elicited increased rCBF in the ACC [Brodmann Area (BA) 24 and 32)] in both groups. Shin and colleagues ${ }^{28}$ also used fMRI to investigate ACC functioning in 16 Vietnam combat veterans (8 with PTSD) using an emotional counting Stroop (ecStroop) paradigm. Subjects were asked to count the number of combat-related, generally negative and neutral words while being scanned. In the comparison of combat related to generally negative words the non-PTSD group showed significant BOLD signal increases in rACC but the PTSD group did not.

\section{Hippocampus}

Shin and colleagues also investigated hippocampal function in 16 firefighters ( 8 with PTSD) using a word stem completion task and PET. ${ }^{29}$ Subjects completed a three-letter word stem with deeply encoded/high recall and shallow encoded/low recall—words encoded during a preceding training session. The PTSD group demonstrated greater rCBF in the hippocampi (bilateral) across conditions. In the main contrast of interest (high vs. low recall) the PTSD group (vs. control group) showed significantly smaller rCBF increases in the left hippocampus, which was primarily driven by relatively elevated $\mathrm{rCBF}$ in the low recall condition. In another study, the same group used emotional facial expressions and fMRI to compare BOLD responses in 13 men with PTSD and 13 trauma-exposed men without PTSD. ${ }^{30}$ The PTSD group showed increased amygdala responses and decreased $\mathrm{mPFC}$ responses to overt fearful (vs. happy) facial expressions. BOLD signal changes in the amygdala in the PTSD subjects were negatively correlated with signal changes in the mPFC. Additionally, BOLD signal changes in the mPFC were inversely correlated with symptom severity (CAPS).

Another group investigated mechanisms of updating working memory in PTSD using $\left[{ }^{15} \mathrm{O}\right] \mathrm{H}_{2} \mathrm{O}$ PET and a variant of the n-back task (detection of trauma-unrelated target words under fixed and variable conditions; only the variable condition required target updating) in 10 patients with PTSD (mostly civilian trauma) compared to 10 healthy subjects. ${ }^{31}$ Functional connectivity analysis during the working memory task revealed increased activation in bilateral $(\mathrm{B} / \mathrm{L})$ inferior parietal lobules and left precentral gyrus, and reduced activation in inferior medial frontal lobe, $\mathrm{B} / \mathrm{L}$ middle frontal gyri, and right inferior temporal gyrus, in the PTSD group relative to the control subjects.

The deactivation or failure of activation of the rACC during the processing of emotional stimuli in PTSD but not traumatized control subjects is consistent with similar findings from symptom provocation studies. This subregion of the ACC has rich connections with the OFC, anterior insula, nucleus 
accumbens, hippocampus and hypothalamus, and brain stem regions and has an outflow to autonomic, visceromotor, and endocrine systems. It has been called the "affective" subdivision on account of its involvement in the assessment of salience of emotional information and the regulation of emotional responses. ${ }^{32}$

\section{Functional Connectivity Analyses}

It is increasingly evident that different brain regions act in concert during the performance of cognitive-emotional tasks. This understanding can be extended to PTSD where symptoms may result from abnormalities in cognitiveemotional processes that reflect dysfunctional networks of neural activity, rather than or in addition to altered activity in specific regions. Investigators have been applying advanced statistical methods to identify interregional brain activity correlations (termed functional connectivity analyses), or the influence of one brain region over another (effective connectivity), in the context of specific tasks. ${ }^{33,34}$

A few recent studies have applied these methods to neuroimaging studies of PTSD. Gilboa and colleagues studied 20 individuals with a history of civilian trauma (10 with PTSD), using symptom provocation (autobiographical traumarelated and neutral scripts) and $\left[{ }^{15} \mathrm{O}\right] \mathrm{H}_{2} \mathrm{O}$ PET. ${ }^{35}$ A multivariate analysis technique (partial least squares) was used to identify brain regions whose activity covaried with two reference ("seed") voxels, one in right PFC (BA 10) and the other in right amygdala (both derived from a preliminary task). The authors reported a significant influence of amygdala activity on activity in the visual cortex, subcallosal gyrus, and anterior cingulate in the PTSD subjects but not in the trauma-exposed controls ("effective connectivity"). They were, however, unable to find support for the failure of inhibition of the ACC over the amygdala.

Lanius and colleagues also used functional connectivity analyses on data gathered during fMRI script-driven symptom provocation experiments in 11 subjects with PTSD on account of sexual abuse/assault or motor vehicle accident (MVA), and 13 trauma-exposed subjects without PTSD. ${ }^{36}$ Comparison of connectivity maps at a right ACC coordinate showed greater correlations in PTSD subjects (vs. controls) in the right posterior cingulate cortex (PCC) (BA 29), right caudate, right parietal lobe (BA 7 and 40), and right occipital lobe (BA 19). Subjects without PTSD had greater correlations (vs. PTSD subjects) in the left superior frontal gyrus (BA 9), left anterior ACC (BA 32), left striatum (caudate), left parietal lobe (BA 40 and 43), and left insula (BA 13).

Functional connectivity methods are still under development and their neurobiological relevance is not yet completely clear. However, they begin to provide a way to investigate functional relationships between brain regions in health and disease. 


\title{
Summary of Functional Neuroimaging Studies in PTSD
}

Thus, several studies examining different cohorts (combat and CSA-related PTSD), different paradigms (symptom provocation vs. cognitive activation), and modalities (fMRI and PET) have demonstrated reduced activation of the mPFC (BA 10 and 11) and ACC (BA 32) in PTSD subjects compared to traumatized controls. ${ }^{14,16-18,28}$ Other studies have reported increased responsivity of the amygdaloid region ${ }^{11,13,26}$ though some have not. ${ }^{14,16,17}$ These findings may reflect dysfunctionality in cortical structures important in the regulation of emotional responses and the process of extinction of fear conditioning ( $\mathrm{mPFC}$ and ACC). They also suggest the presence of hyperresponsivity of subcortical structures, specifically the amygdaloid region that is involved in the assessment of stimulus salience, stimulus reinforcer associations, and vigilance. These regions are richly interconnected. The failure of higher regulatory structures to modulate the activity of the amygdala and related subcortical structures is thought to be related to the exaggerated emotional responses seen in PTSD. Thus some evidence from human neuroimaging studies supports the extension of animal research findings of functional connectivity between medial prefrontal cortical structures and the amygdala.

As discussed above, while these findings are important, several factors need to be considered. These findings do not readily lend themselves to improving our understanding of significant manifestations of PTSD, such as intrusive thoughts and memories, avoidance and numbing, significant mood and substance-related comorbidity, and chronicity of PTSD. There is also insufficient evidence to provide clarity to whether or not these mechanisms are specific to PTSD (i.e., differentiated from effects of trauma, comorbidity, or are shared across other anxiety disorders). Other relevant mechanisms that may assist in understanding the complex phenomenology of PTSD need to be explored, such as the phenomena of conditioning, habituation, stimulus generalization, and (resistance to) extinction; cognitive-emotional modulation (appraisal and reappraisal); and social and self-related emotional processing. The relevance of studying these phenomena in PTSD will be discussed in more details in the following section, which includes a selective review of neuroimaging research that focuses on fear conditioning, cognitive-emotional interactions, and self-related and social-emotional processing in healthy humans.

\section{Emotional Responses in Healthy Subjects Relevant to PTSD}

\author{
Neuroimaging Studies of Threat-Related Processing (Fear Conditioning, \\ Habituation, and Extinction)
}

Elegant studies using the fear conditioning paradigm in rats over the past two decades have helped outline a specialized threat-related neural network 
that involves several functionally connected regions including the subregions of the PFC, the amygdala, and the hippocampus. Evidence also exists to suggest the existence of two broad pathways in the processing of threat-related emotion - a subcortical "fast" pathway that transmits stimulus characteristics rapidly but with less specificity and a cortical "slow" pathway that involves nuanced cognitive processing of stimulus characteristics. ${ }^{1}$ Animal studies have identified the amygdaloid complex (specifically the central nucleus and lateral and basolateral nuclei) as a crucial substrate in the formation of stimulus response associations involved in the fear conditioning response as well as aversive learning. The similarity between the behavioral and autonomic and neuroendocrine manifestations of the fear conditioning response and aspects of anxiety and PTSD symptomatology (hyperarousal symptoms) has inspired several investigators to study this phenomenon in humans.

Specifically, several investigators have studied the fear conditioning paradigm in healthy humans using fMRI or PET. ${ }^{37}$ In a novel $\left[{ }^{15} \mathrm{O}\right] \mathrm{H}_{2} \mathrm{O}$ PET study, Morris and colleagues used overtly presented and masked faces as the conditioned stimulus (CS) and a 1-s 100-dB burst of white noise as the unconditioned stimulus (US) in 10 healthy right- handed male subjects and demonstrated amygdala activation during CS + trials (CS coupled with aversive US) but not during CS - trials (CS not associated with US). They also noted that the automatic or subconscious presentation of the CS (masked CS+ minus masked CS-) activated the right amygdala whereas conscious presentation of the CS (unmasked CS+ minus unmasked CS-) activated the left amygdala suggesting a differential lateralized response to automatic or implicit versus conscious or explicit processing of these stimuli. ${ }^{38}$ These findings provide direct evidence for the role of the amygdala in emotional learning (of behaviorally significant stimuli) and demonstrate that this learning can occur even in the absence of conscious perception of such stimuli. Thus it is conceivable that hyperresponsivity of the amygdala that has been hypothesized in PTSD may lead to exaggerated emotional responsivity to behaviorally significant (threat related) stimuli. This remains to be demonstrated in PTSD.

Two fMRI studies using aversive delay conditioning paradigms, showed that the CS+ was associated with a greater activation of the ACC and amygdala, and additionally found a CS+-related signal decrement over time suggesting habituation of this response. ${ }^{39,40}$ Another event-related fMRI(er-fMRI) study involved a trace conditioning paradigm and demonstrated anterior hippocampal, amygdala, and ACC activation with similar temporal decrement. ${ }^{41}$ A recent study using er-fMRI compared the delay and trace conditioning methods. Conditions were $\mathrm{CS}+, \mathrm{CS}-$, and $\mathrm{CS}_{10}$ (US and CS separated by a 10-s trace interval); pain ratings, skin conductance response (SCR), and shock expectancy were recorded. Overlapping patterns of activation in the ACC, medial thalamus, and visual cortex were seen during delay and trace procedures, with additional recruitment of the hippocampus during trace conditioning. ${ }^{42}$ These neuroimaging studies of fear conditioning provide evidence for the involve- 
ment of the ACC and amygdala in the acquisition of fear conditioning in humans. As noted, both fear conditioning and the involved brain regions are implicated in the cued hyperarousal symptoms in PTSD.

Habituation is a process of rapid attenuation of a neural response as a function of a repeatedly presented stimulus while extinction refers to a reduction and disappearance of a conditioned response on account of learning about a new stimulus-response association (i.e., the CS is no longer associated with the associated US). These are considered adaptive processes for organisms as they provide the organism with flexibility to reallocate critical resources to threat-related stimuli in a constantly changing environment. The failure of habituation to trauma-related stimuli and/or the failure of extinction have been hypothesized to contribute to the development or maintenance of PTSD following exposure to trauma (conditioning). These phenomena have been extensively studied in animals and behaviorally in humans but these processes have only recently been the subject of neuroimaging investigations.

Two earlier MRI studies involving emotionally expressive faces presented both overtly as well as in a masked manner (fearful or happy faces masked with a neutral face such that subjects consciously perceive only the neutral face) revealed that the amygdala was activated to emotionally valenced faces and this response rapidly habituates regardless of the mode of presentation (overt or masked). ${ }^{43,44}$ Authors of another study used repeated presentations of complex visual stimuli (aversive and neutral video clips) in conjunction with PET and reported that just two repetitions of the stimuli were associated with reduction in $\mathrm{rCBF}$ in the secondary visual cortices and the right medial temporal cortex including the amygdala and hippocampus though there was no difference in the rates of habituation between the aversive and neutral stimuli. ${ }^{45}$ This was followed by an fMRI study, of repeated presentations of fearful and neutral faces, which revealed signal decrement in the right amygdala and hippocampus as well as bilateral medial/inferior temporal cortex though again this finding was not specific to the expression condition (fearful vs. neutral). ${ }^{46} \mathrm{In}$ another fMRI study, fearful and happy faces were repeatedly presented in two 2 -min runs to eight right-handed healthy male subjects. Significant fMRI signal decrement was observed in the left dorsolateral prefrontal cortex (dlPFC) and premotor cortex, and the right amygdala. Only the left dlPFC showed differential habituation to the valence of the faces with greater habituation to happy than fearful faces, which possibly reflects differential responses of prefrontal versus subcortical structures to threat-related stimuli. Additionally, the right amygdala exhibited greater habituation to emotionally valenced stimuli (than the left) while the left amygdala responded significantly more to negatively versus positively valenced stimuli (relative to the right). ${ }^{47}$ Our laboratory has also demonstrated $\mathrm{rACC}$ habituation with repeated emotional picture (aversive minus neutral/blank) presentation. ${ }^{48}$

These studies provide evidence for habituation in the dlPFC, ACC, and the amygdala with some evidence for differential habituation in prefrontal versus 
subcortical regions to threat-related stimuli, as well as lateralized specialization of rapid versus sustained response to threat stimuli. ${ }^{47}$ Interestingly, the only study that has specifically addressed the time course of amygdala responses to trauma cues (trauma-relevant words) in PTSD patients and healthy controls found an increased left amygdala response to trauma-relevant negative versus neutral stimuli in the first two but not last two runs. This response correlated with the symptom severity (CAPS total score). However, while sensitization to nontrauma negative words was seen in the PTSD group, failure of habituation to trauma-related words was not seen. ${ }^{24}$

The process of extinction has also been the subject of recent neuroimaging studies. Phelps and colleagues used a simple discrimination, partial reinforcement fear conditioning paradigm in a trial-related fMRI design in 11 righthanded subjects. ${ }^{49}$ Colored squares were used for CS+ and CS- (blue and yellow) and US was a mild wrist shock. The study was conducted in three phases (acquisition, day 1 extinction, and day 2 extinction). Skin conductance responses were recorded. fMRI scanning occurred during all phases (two runs for each learning phase). CS were presented for 4 seconds with a 12-second inter-trial interval (ITI). Group analyses were conducted for each phase (CS+ versus $\mathrm{CS}-$ ) followed by hypothesis-driven region of interest (ROI) analyses of $\mathrm{mPFC}$ (functional ROI) and amygdala (anatomical ROI). The authors reported that right amygdala activation predicted the conditioned response (CR) in the early acquisition (positive correlation) and day 1 extinction phase (negative). The vmPFC (the subgenual anterior cingulate ROI) response positively correlated with the CR magnitude during day 2 extinction. These findings appear to be consistent with those of animal research that implicates the amygdala in acquisition and extinction and the vmPFC in the retention of extinction learning process, and are intriguing in light of evidence of altered connectivity between medial frontal regions and amygdala in PTSD.

In another study, an olfactory aversive conditioning and extinction paradigm was used in an er-fMRI design. The authors reported that discrete regions of the rostral and caudal OFC and lateral amygdala were preferentially activated during the extinction process. Their findings appear consistent with animal literature that implicates the vmPFC and amygdala in the extinction-related processes. ${ }^{50-53}$ They also demonstrated that the CS+ retains access to representations of the US in distinct regions of the ventral PFC even as the extinction learning process proceeds (which supports the notion that extinction involves formation of new associations rather than erasure of the old ones).

Thus, the evidence from human neuroimaging studies discussed above implicates subregions of the MPFC and OFC, subdivisions of the ACC, the extended amygdala, the hippocampus, and nuclei of the thalamus in the processes of fear conditioning, habituation, and extinction. The fear conditioning paradigm and the associated phenomena of habituation and extinction (or failure of these processes) are relevant to aspects of psychopathological states, such as PTSD and phobic states. The neuroimaging studies of these processes in healthy 
humans provide a background for extending these studies to patients with PTSD and other anxiety disorders

\section{Cognitive-Emotional Interactions: Appraisal, Reappraisal, and Emotional Regulation}

The reciprocal relationship between cognition and emotion is receiving increased attention on account of the importance of these processes in understanding human decision making, emotion regulation, and behavior. These processes have significant implications for the study of psychopathological states, such as PTSD where dysfunctional attributional biases and emotional dysregulation and resultant behavioral dysfunction are observed. For purposes of this discussion, cognitive-emotional interactions refers to component processes of appraisal, reappraisal, and emotional regulation

\section{Cognitive Appraisal of Emotions}

Cognitive appraisal broadly refers to the interpretation of stimuli. An increasing number of neuroimaging studies in healthy subjects are providing evidence that cognitive processes can modulate subjective emotional responses, which is reflected in the activity of emotion processing areas. Even the simple process of labeling or rating an emotion has been shown to reduce the activity in structures that are responsive when the emotional stimulus is passively viewed or experienced.

Studies have examined the effects of cognitive tasks on emotional processing and the areas that subserve them in healthy subjects. Hariri and his colleagues investigated the effects of a cognitive task on emotional processing in healthy subjects by comparing their BOLD response while performing three different tasks (match, label, and control). In the match task, subjects were asked to match the effect of one of two faces to that of a simultaneously presented target face (angry or fearful) whereas in the label task they were to identify the effect of a target face by choosing one of two simultaneously presented linguistic labels (angry or afraid). The control task involved matching a target shape. Matching was associated with increased activation in both the right and left amygdala whereas linguistically labeling the expression was associated with a decreased activation in the amygdala. Additionally, right PFC activity was inversely correlated with left amygdala activity. The authors interpreted these findings as evidence in support of prefrontal cortical structures being the neural substrate for the cognitive modulation of emotion via the process of interpretation and labeling. ${ }^{54}$ This finding has been replicated using threatening and fearful pictures as well. ${ }^{55}$

We examined rCBF response in healthy subjects comparing an appraisal to a passive viewing condition. ${ }^{56}$ Subjects saw aversive and neutral pictures from 
the international affective picture system (IAPS), ${ }^{57}$ while they performed a passive viewing (PSVW) and appraisal ("rating"-RTNG) task. During PSVW, for aversive minus neutral pictures, subjects activated foci in the area of the right insula/amygdala and left insula. RTNG was associated with increased activation of the dorsal medial prefrontal cortex (dmPFC) and the ACC. RTNG resulted in reduction in the intensity of sadness and reduced activation of the right insula/amygdala and left insula compared to PSVW. These findings demonstrate the involvement of the dmPFC and ACC in cognitive tasks (appraisal) and suggest modulating effects of these structures on emotion-related structures, such as the amygdala and insula. These findings extend findings from animal studies that have demonstrated the inhibitory influence of the mPFC over the amygdala. ${ }^{58}$

These studies provide impetus to study cognitive appraisal processes in PTSD using similar paradigms. Several studies have identified that the peritraumatic appraisal of stressful events and even the posttraumatic appraisal (of PTSD symptoms) as threatening constitute a significant risk factor for developing and maintaining PTSD. ${ }^{58}$ Thus, characterizing dysfunctional appraisal mechanisms in PTSD and their underlying neural substrate may result in the availability of biological markers of a hypothesized overresponsive threat detection system.

\section{Cognitive Modulation of Emotions-Reappraisal and Emotional Regulation}

Cognitive reappraisal can be understood as a form of cognitive regulation that involves reinterpreting the meaning of a stimulus to change one's emotional response to it. ${ }^{60}$ Recently, several groups have investigated the effects of voluntary cognitive manipulation of experienced emotion using functional neuroimaging methods. This line of work is highly relevant to the study of PTSD, where emotional dysregulation is a predominant feature. It is also of much interest in the investigation of brain mechanisms of cognitive behavioral therapy, an effective treatment for some patients with PTSD.

Gross and colleagues, have proposed two contrasting mechanisms by means of which humans may regulate their emotions. In cognitive regulation (reappraisal), there is an active attending to and/or reinterpretation of negative emotion (such as positive reframing) that reduces the emotional response and might decrease physiological arousal but does not impair memory, which the authors suggest is an adaptive mechanism. Behavioral regulation (suppression) of negative emotions suppresses emotionally expressive behavior but does not dampen unpleasant experience, worsens memory, and increases sympathetic nervous system activation. ${ }^{60}$ While this work clearly does not explain the entire complex phenomenon of cognitive-emotional interaction, it gives us a 
valuable place to start. Ochsner and colleagues have extended this concept to the neuroimaging environment. They used an er-fMRI design and aversive IAPS pictures to study healthy female subjects who were asked to Attend (be aware of feelings elicited by the picture) or to Reappraise (reinterpret the picture so that it no longer elicits a negative emotional response) while being scanned. ${ }^{61}$ Reappraisal of highly negative scenes was successful. Reappraising (vs. attending) was associated with increased activation of the dorsal and ventral left lateral prefronal cortex (IPFC), dmPFC, left temporal pole, right supramarginal gyrus (SMG), and left lateral occipital cortex. Attending (vs. reappraising) was associated with increased activity in the right amygdala, left posterior insula, left medial orbitofrontal cortex (OFC), right medial occipital cortex, and right inferior parietal cortex. Greater activation in the right ACC and SMG correlated with greater decreases in negative affect (greater reappraisal success); left ventral PFC activation during reappraisal was inversely correlated with activity in the amygdala. Effective reappraisal resulted in increased activation in $\mathrm{PFC}$ and $\mathrm{mPFC}$, regions implicated in working memory, cognitive control, and self-monitoring, and in decreased activation of medial OFC and amygdala, regions implicated in emotion processing.

In a follow-up to this study, Ochsner and colleagues using an emotion regulation paradigm and fMRI, demonstrated that both voluntary up- and downregulation of negative emotion recruits prefrontal and ACC regions. Amygdala activation was modulated up or down depending on the regulatory goal. Upregulation uniquely recruited right lateral and orbital PFC. Self (internal)-focused regulation recruited $\mathrm{MPFC}$ regions whereas situation (external)-focused regulation recruited lateral PFC regions. ${ }^{62}$ Using a similar paradigm, Phan and colleagues showed highly aversive and arousing pictures from the IAPS to healthy subjects, who were instructed to either "maintain" (feel naturally) or "suppress" (by positive reframing or rationalizing) negative affect. ${ }^{63}$ Successful reduction of negative affect was associated with increasing activation of dmPFC, dorsal ACC, dIPFC, lateral OFC, and ventrolateral PFC/inferior frontal gyrus, and with decreasing activity in the left nucleus accumbens, left lateral PFC, and left extended amygdala. Additionally, right dorsal ACC, right anterior insula, bilateral dlPFC, and bilateral ventrolateral PFC activity inversely correlated with the intensity of negative affect.

These studies are providing additional evidence for the existence of emotion regulatory (including $\mathrm{LPFC}$, dmPFC, SMG, and ACC) and emotionally responsive regions (including amygdala, insula, $\mathrm{mOFC}$ ) in the human brain. The observed difficulty that patients with PTSD to cognitively regulate their emotions can be hypothesized to be a result of dysfunctional cognitive-emotional processes (such as cognitive appraisal and reappraisal) subserved by some of these regions. The therapeutic mechanisms of cognitive behavioral therapy in PTSD may also be related to these processes and structures. There is therefore a need to extend these innovative paradigms to the study of PTSD. 


\section{Self-Relatedness and Social-Emotional Processing}

The tendency to interpret or perceive stimuli as self-relevant are of specific interest in PTSD given that core manifestations of PTSD include feelings of threat and guilt, difficulties in interpersonal and social functioning, and the observation that interpersonal trauma results in the highest rates of PTSD. ${ }^{64}$ Healthy social functioning is pivotal to the survival of humans and their progeny and serves a protective function with regard to stressors and disease. Thus it makes intuitive sense that this important function be subserved by dedicated neural resources for the processing of social stimuli. Primate and human lesions studies have implicated the mPFC, OFC, superior temporal sulcus, amygdala, and other regions in processing social and related stimuli.

We investigated the concept of self-relatedness in the context of emotional processing in two studies. In the first study, we used aversive, positive, and neutral IAPS pictures in a trial-related fMRI design to compare the neural substrates underlying the assessment of the emotional intensity of the pictures versus the self-relatedness of their content, in healthy, right-handed volunteers. ${ }^{65}$ Individualized subjective ratings over these two dimensions (obtained postscan) were correlated with brain activity in a parametric factorial analysis. The appraisal of self-relatedness specifically engaged the $\mathrm{MPFC}$ and recruited the dmPFC and insula as the extent of self-relatedness increased. On the other hand, the amygdala activation was specific to affective judgment of emotional intensity. Both increasing emotional intensity and self-relatedness activated the nucleus accumbens. These findings suggest that appraisal of self-relatedness specifically recruits the mPFC, a region relevant to the symptomatology and possibly pathophysiology of PTSD

In a recent study we extended our investigation of the neural substrates of emotion to the processing of social versus nonsocial stimuli in 12 healthy, right-handed volunteers (Britton et al., in press). In a novel paradigm, subjects viewed short video segments that evoked positive or negative emotions that were categorized as "social" (humor, sadness) or "nonsocial" (appetite, physical disgust). Following the video, static frames extracted from the video were viewed for $30 \mathrm{~s}$ to help subjects maintain the emotions evoked by the video clip; during this period fMRI images were acquired. Nonsocial and socialemotional experiences resulted in partially overlapping but somewhat separate neural patterns. Social positive and social negative conditions activated amygdala/SLEA, superior temporal gyrus, hippocampus, and posterior cingulate, whereas nonsocial positive and nonsocial negative conditions activated insula and visual cortex. Additional activations depended on both social context and valence: amygdala (nonsocial negative); ACC (nonsocial positive and social negative); and OFC and nucleus accumbens (social positive). These findings highlight the importance of sociality in human emotions and provide evidence for the need for attention to this aspect while designing neuroimaging studies of emotion. They also highlight the involvement of structures, such as the PFC, 
ACC, and amygdala in social-emotional processing; structures that are also implicated in PTSD.

\section{Functional Neuroanatomy of Neuroendocrine Stress Regulation in PTSD}

To fully understand the development of pathophysiology of PTSD, it is important that we attempt to integrate functional neuroanatomical findings with those from neurochemical, neurophysiological, and neuroendocrinological studies in PTSD. Indeed, neurobiological research over the past few decades has revealed consistent abnormalities in stress systems, such as catecholaminergic and limbic hypothalamic pituitary adrenal (LHPA) axis systems in PTSD. ${ }^{65,66}$ Concurrently, it is also becoming clear that stress hormones, such as cortisol, play an important role in cognitive and emotional processes in health, mood, and anxiety disorders. ${ }^{68}$ However, much remains to be known about the functional neuroanatomy of these stress systems and their abnormalities especially in psychopathological states, such as PTSD. Few neuroimaging studies have incorporated these parameters on account of inherent difficulties in conducting such a complex investigation in the scanning environment.

Several investigators are attempting to bridge that gap in the areas of mood and trauma-related disorders. Ottowitz and colleagues ${ }^{69}$ with the objective of investigating the neural correlates of relevance to adrenocorticotropic hormone (ACTH) and cortisol regulation, combined a SPECT study of induced sadness in eight healthy women with a linear regression analysis of ACTH and cortisol levels during mood induction. Within-group analysis of the sadness condition with $\mathrm{ACTH}$ as the covariate of interest showed a significant positive correlation between $\mathrm{ACTH}$ and $\mathrm{rCBF}$ in the left anterior cingulate and right insular cortices and a significant negative correlation between $\mathrm{ACTH}$ and $\mathrm{rCBF}$ at the junction of the right caudal OFC and vmPFC. The same analysis with cortisol as the covariate of interest showed a significant positive correlation between cortisol and $\mathrm{rCBF}$ in the left insula.

Drevets and colleagues, ${ }^{70}$ using $18 \mathrm{~F}$-fluorodeoxyglucose PET, measured regional amygdala and hippocampal glucose metabolism in patients with familial pure depressive disease (FPDD), bipolar disorder depressed (BD-D), depressive spectrum disorder (DSD), and healthy controls. Left amygdala metabolism was significantly increased in both the FPDD and BD-D groups relative to the control group. Left amygdala metabolism was also positively correlated with stressed plasma cortisol levels (drawn during the scanning procedure) in both the FPDD and the BD-D groups. This finding may reflect abnormalities in the feedback mechanisms for cortisol possibly mediated by the influence of the amygdala on central stress systems (CRF).

In a resting state study in PTSD, Bonne and colleagues compared 11 subjects with PTSD to 17 trauma-exposed subjects without PTSD and 17 nontraumatized healthy controls using ${ }^{99} \mathrm{~m}_{\mathrm{c}}$-HMPAO SPECT 6 months after the trauma. ${ }^{71}$ They found increased regional blood flow in the cerebellum 
in the PTSD group, compared to both control groups. They also found higher rCBF in right precentral, superior temporal, and fusiform gyri in PTSD compared to healthy controls. Furthermore, cerebellar and extrastriate rCBF positively correlated with measures of depression and PTSD severity. Cortisol level in PTSD was negatively correlated with medial temporal lobe perfusion. Anterior cingulate perfusion and cortisol levels were positively correlated in PTSD and negatively correlated in trauma survivors without PTSD.

Recently, we conducted a $\left[{ }^{15} \mathrm{O}\right] \mathrm{H}_{2} \mathrm{O}$ PET study of a series of emotional challenges (aversive pictures and autobiographic narratives) in 16 combat PTSD patients, 15 combat controls, and 14 noncombat controls. Plasma ACTH and cortisol were measured $2 \mathrm{~min}$ before each stimulus presentation and 5 min after each scan thus allowing us to capture dynamic modulation of neural activity in coordination with the HPA axis function in a within- subjects design. Combatexposed groups demonstrated ACTH responses to the first trauma script, but not to aversive pictures. Voxel-wise analyses showed ACTH responses covaried with $\mathrm{rCBF}$ in $\mathrm{rACC}$ and right insula in PTSD patients and rostral anterior cingulate and $\mathrm{dmPFC}$ in combat controls. These findings suggest involvement of insula, dmPFC, and rostral anterior cingulate in HPA axis responses to trauma-related stimuli. Prestimulus plasma cortisol level covaried with rCBF in subgenual ACC in PTSD patients and rostral anterior cingulate in combat controls. This suggests that rACC may be a site of modulation by circulating cortisol in trauma-exposed subjects. Differential patterns of covariation between combat veterans with and without PTSD implicate dmPFC and rostral anterior cingulate as areas of dysregulation of HPA axis responses in PTSD (King et al., unpublished).

Taken together, these findings suggest that specific prefrontal cortical regions, the ACC, the insula, and the amygdala are intimately involved in the activation and modulation of stress response. The same regions are implicated in emotional processing in general and social emotions in particular and in the symptomatology of PTSD. Activation of neuroendocrine stress response in turn appears to have a modulating activity in these areas.

\section{SUMMARY AND FUTURE DIRECTIONS}

Neuroimaging studies of PTSD over the past decade have been important and informative yet are unable to fully capture the complexity of PTSD. The evidence so far in PTSD consists of regionally specific, impaired blood flow patterns suggesting impaired functioning in subregions of the $\mathrm{mPFC}$ and anterior cingulate regions, ${ }^{15-17,28}$ and increased/altered blood flow patterns suggesting increased responsivity of the extended amygdala and insula regions. ${ }^{11,13,26}$ Findings are not always consistent and may be influenced by several methodological issues, such as small sample sizes, heterogeneous populations, and varying imaging methods that limit broad generalization. 
Several lines of neuroimaging research in the cognitive neuroscience addressing cognitive-emotional interactions (threat processing, appraisal, reappraisal, emotion regulation; the concept of self-relatedness and social emotional processing) are implicating the same regions identified in prior studies of PTSD (such as the mPFC, ACC, amygdala). This is hardly surprising considering the cognitive-emotional bases of PTSD symptoms. Additionally, studies are investigating the neural control of stress-related systems and their interactions with cognitive-emotional processes in health and in psychopathological states. These exciting lines of research promise to further improve our understanding of processes and structures involved in the processing of stressful events in health and disease states.

There is therefore a clear need to move toward the next decade of progress in PTSD research by incorporating the innovative paradigms currently being developed for the study of emotion, cognitive-emotional processing, and social cognitive neuroscience, to the study of PTSD. These include neuroimaging studies of fear conditioning, habituation, and extinction as well as studies investigating self-related and social emotional processing and cognitive appraisal and reappraisal mechanisms. There is also a need for integrating different lines of inquiry, including genetic, neurochemical/receptor, HPA axis, and blood flow parameters in PTSD. This research holds the exciting promise of helping to identify neurobiological factors that may confer vulnerability or resilience to PTSD and adaptive from dysfunctional processes and offer meaningful clues to the pathophysiology of PTSD. This progress will be essential for the future development of effective prevention and treatment strategies for this disorder.

\section{REFERENCES}

1. LeDoux, J.E. 2000. Emotion circuits in the brain. Annu. Rev. Neurosci. 23: 155184.

2. Phan, K.L. et al. 2002. Functional neuroanatomy of emotion: a meta-analysis of emotion activation studies in PET and fMRI. Neuroimage 16: 331-348.

3. RAUCH, S.L. \& L.M. SHIN. 1997. Functional neuroimaging studies in posttraumatic stress disorder. Ann. N.Y. Acad. Sci. 821: 83-98.

4. Pitman, R.K., L.M. Shin \& S.L. RaUCH. 2001. Investigating the pathogenesis of posttraumatic stress disorder with neuroimaging. J. Clin. Psychiatry 62: 47-54.

5. Liberzon, I. \& K.L. Phan. 2003. Brain-imaging studies of posttraumatic stress disorder. CNS Spectrums 8: 641-650.

6. Pitman, R.K., S.P. OrR \& G.S. Steketee. 1989. Psychophysiological investigations of posttraumatic stress disorder imagery. Psychopharmacol. Bull. 25: 426-431.

7. Pitman, R.K. et al. 1990. Psychophysiologic responses to combat imagery of Vietnam veterans with posttraumatic stress disorder versus other anxiety disorders. J. Abnorm. Psychol. 99: 49-54.

8. Shalev, A.Y. et al. 1997. Psychophysiologic assessment of mental imagery of stressful events in Israeli civilian posttraumatic stress disorder patients. Compr. Psychiatry 38: 269-273. 
9. LiBERzon, I. et al. 2000. Limbic activation and psychophysiologic responses to aversive visual stimuli. Interaction with cognitive task. Neuropsychopharmacology 23: 508-516.

10. Pitman, R.K. \& S.P. ORR. 1990. Twenty-four hour urinary cortisol and catecholamine excretion in combat-related posttraumatic stress disorder. Biol. Psychiatry 27: 245-247.

11. RAUCH, S.L. et al. 1996. A symptom provocation study of posttraumatic stress disorder using positron emission tomography and script-driven imagery. Arch. Gen. Psychiatry 53: 380-387.

12. SHIN, L.M. et al. 1997. Visual imagery and perception in posttraumatic stress disorder. A positron emission tomographic investigation. Arch. Gen. Psychiatry 54: 233-241.

13. LIBERzon, I. et al. 1999. Brain activation in PTSD in response to trauma-related stimuli. Biol. Psychiatry 45: 817-826.

14. BREMNER, J.D. et al. 1999. Neural correlates of exposure to traumatic pictures and sound in Vietnam combat veterans with and without posttraumatic stress disorder: a positron emission tomography study. Biol. Psychiatry 45: 806-816.

15. BREMNER, J.D. et al. 1999. Neural correlates of memories of childhood sexual abuse in women with and without posttraumatic stress disorder. Am. J. Psychiatry 156: 1787-1795.

16. SHIN, L.M. et al. 1999. Regional cerebral blood flow during script-driven imagery in childhood sexual abuse-related PTSD: a PET investigation. Am. J. Psychiatry 156: $575-584$.

17. LANIUS, R.A. et al. 2001. Neural correlates of traumatic memories in posttraumatic stress disorder: a functional MRI investigation. Am. J. Psychiatry 158: 19201922.

18. LANIUS, R.A. et al. 2003. Recall of emotional states in posttraumatic stress disorder: an fMRI investigation. Biol. Psychiatry 53: 204-210.

19. Hendler, T., P. Rotshtein \& U. Hadar. 2001. Emotion-perception interplay in the visual cortex: "the eyes follow the heart". Cell. Mol. Neurobiol. 21: 733-752.

20. SHIN, L.M. et al. 2004. Regional cerebral blood flow in the amygdala and medial prefrontal cortex during traumatic imagery in male and female Vietnam veterans with PTSD. Arch. Gen. Psychiatry 61: 168-176.

21. BRITTON, J.C. et al. 2005. Corticolimbic blood flow in posttraumatic stress disorder during script-driven imagery. Biol. Psychiatry 57: 832-840.

22. Osuch, E.A. et al. 2001. Regional cerebral blood flow correlated with flashback intensity in patients with posttraumatic stress disorder. Biol. Psychiatry 50: 246253.

23. LANiUs, R.A. et al. 2002. Brain activation during script-driven imagery induced dissociative responses in PTSD: a functional magnetic resonance imaging investigation. Biol. Psychiatry 52: 305-311.

24. Protopopescu, X. et al. 2005. Differential time courses and specificity of amygdala activity in posttraumatic stress disorder subjects and normal control subjects. Biol. Psychiatry 57: 464-473.

25. Semple, W.E. et al. 1996. Attention and regional cerebral blood flow in posttraumatic stress disorder patients with substance abuse histories. Psychiatry Res. 67: $17-28$.

26. RAUCH, S.L. et al. 2000. Exaggerated amygdala response to masked facial stimuli in posttraumatic stress disorder: a functional MRI study. Biol. Psychiatry 47: 769-776. 
27. BRemner, J.D. et al. 2004. Neural correlates of the classic color and emotional Stroop in women with abuse-related posttraumatic stress disorder. Biol. Psychiatry 55: 612-620.

28. SHIN, L.M. et al. 2001. An fMRI study of anterior cingulate function in posttraumatic stress disorder. Biol. Psychiatry 50: 932-942.

29. SHIN, L.M. et al. 2004. Hippocampal function in posttraumatic stress disorder. Hippocampus 14: 292-300.

30. SHIN, L.M. et al. 2005. A functional magnetic resonance imaging study of amygdala and medial prefrontal cortex responses to overtly presented fearful faces in posttraumatic stress disorder. Arch. Gen. Psychiatry 62: 273-281.

31. SHAw, M.E. et al. 2002. Abnormal functional connectivity in posttraumatic stress disorder. Neuroimage 15: 661-674.

32. Bush, G., P. LuU \& M.I. Posner. 2000. Cognitive and emotional influences in anterior cingulate cortex. Trends Cogn. Sci. 4: 215-222.

33. Friston, K.J. et al. 1993. Functional connectivity: the principal-component analysis of large (PET) data sets. J. Cereb. Blood Flow Metab. 13: 5-14.

34. FRISTON, K.J. et al. 1996. Functional topography: multidimensional scaling and functional connectivity in the brain. Cereb Cortex 6: 156-164.

35. GilboA, A. et al. 2004. Functional connectivity of the prefrontal cortex and the amygdala in posttraumatic stress disorder. Biol. Psychiatry 55: 263-272.

36. LANIUS, R.A. et al. 2004. The nature of traumatic memories: a 4-T FMRI functional connectivity analysis. Am. J. Psychiatry 161: 36-44.

37. Buchel, C. \& R.J. Dolan. 2000. Classical fear conditioning in functional neuroimaging. Curr. Opin. Neurobiol. 10: 219-223.

38. Morris, J.S., A. OHMAN \& R.J. Dolan. 1998. Conscious and unconscious emotional learning in the human amygdala. Nature 393: 467-470.

39. LABAR, K.S. et al. 1998. Human amygdala activation during conditioned fear acquisition and extinction: a mixed-trial fMRI study. Neuron 20: 937945.

40. BuCHEL, C. et al. 1998. Brain systems mediating aversive conditioning: an eventrelated fMRI study. Neuron 20: 947-957.

41. BuCHEL, C. et al. 1999. Amygdala-hippocampal involvement in human aversive trace conditioning revealed through event-related functional magnetic resonance imaging. J. Neurosci. 19: 10869-10876.

42. KNIGHT, D.C. et al. 2004. Neural substrates mediating human delay and trace fear conditioning. J. Neurosci. 24: 218-228.

43. BREITER, H.C. et al. 1996. Response and habituation of the human amygdala during visual processing of facial expression. Neuron 17: 875-887.

44. Whalen, P.J. et al. 1998. Masked presentations of emotional facial expressions modulate amygdala activity without explicit knowledge. J. Neurosci. 18: 411418.

45. FISCHER, H. et al. 2000. Brain representation of habituation to repeated complex visual stimulation studied with PET. Neuroreport 11: 123-126.

46. FiSCHER, H. et al. 2003. Brain habituation during repeated exposure to fearful and neutral faces: a functional MRI study. Brain Res. Bull. 59: 387-392.

47. WRIGHT, C.I. et al. 2001. Differential prefrontal cortex and amygdala habituation to repeatedly presented emotional stimuli. Neuroreport 12: 379-383.

48. Phan, K.L. et al. 2003. Habituation of rostral anterior cingulate cortex to repeated emotionally salient pictures. Neuropsychopharmacology 28: $1344-$ 1350. 
49. Phelps, E.A., M.R. Delgado, K.I. Nearing, et al. 2004. Extinction learning in humans: role of the amygdala and umPFC. Neuron 43: 897-905.

50. Morgan, M.A., L.M. Romanski \& J.E. LeDoux. 1993. Extinction of emotional learning: contribution of medial prefrontal cortex. Neurosci. Lett. 163: 109113.

51. Morgan, M.A., J. Schulkin \& J.E. LeDouX. 2003. Ventral medial prefrontal cortex and emotional perseveration: the memory for prior extinction training. Behav. Brain Res. 146: 121-130.

52. Morgan, M.A. \& J.E. LeDoux. 1995. Differential contribution of dorsal and ventral medial prefrontal cortex to the acquisition and extinction of conditioned fear in rats. Behav. Neurosci. 109: 681-688.

53. Falls, W.A., M.J. Miserendino \& M. Davis. 1992. Extinction of fear-potentiated startle: blockade by infusion of an NMDA antagonist into the amygdala. J. Neurosci. 12: 854-863.

54. Hariri, A.R., S.Y. Bookheimer \& J.C. MAzziotta. 2000. Modulating emotional responses: effects of a neocortical network on the limbic system. Neuroreport 11: $43-48$.

55. HARIRI, A.R. et al. 2003. Neocortical modulation of the amygdala response to fearful stimuli. Biol. Psychiatry 53: 494-501.

56. TAYLOR, S.F. et al. 2003. Subjective rating of emotionally salient stimuli modulates neural activity. Neuroimage 18: 650-659.

57. LANG, P.J., M.M. BRADLEY \& B.N. CUTHBERT. 1997 International Affective Picture System (IAPS): Technical Manual and Affective Ratings. NIMH Center for the Study of Emotion and Attention, University of Florida. Gainesville, FL.

58. Rosenkranz, J.A. \& A.A. Grace. 2002. Cellular mechanisms of infralimbic and prelimbic prefrontal cortical inhibition and dopaminergic modulation of basolateral amygdala neurons in vivo. J. Neurosci. 22: 324-337.

59. MCNALLY, R.J. 2003. Psychological mechanisms in acute response to trauma. Biol. Psychiatry 53: 779-788.

60. Gross, J.J. 2002. Emotion regulation: affective, cognitive, and social consequences. Psychophysiology 39: 281-291.

61. OCHSNER, K.N. et al. 2002. Rethinking feelings: an FMRI study of the cognitive regulation of emotion. J. Cogn. Neurosci. 14: 1215-1229.

62. OCHSNER, K.N. et al. 2004. For better or for worse: neural systems supporting the cognitive down- and up-regulation of negative emotion. Neuroimage 23: 483-499.

63. PHAN, K.L. et al. 2005. Neural substrates for voluntary suppression of negative affect: a functional magnetic resonance imaging study. Biol. Psychiatry 57: 210219.

64. Kessler, R.C. et al. 1995. Posttraumatic stress disorder in the National Comorbidity Survey. Arch. Gen. Psychiatry 52: 1048-1060.

65. Phan, K.L. et al. 2004. Neural correlates of individual ratings of emotional salience: a trial-related fMRI study. Neuroimage 21: 768-780.

66. LibERzon, I. et al. 1999. Neuroendocrine and psychophysiologic responses in PTSD: a symptom provocation study. Neuropsychopharmacology 21: 40-50.

67. Newport, D.J. \& C.B. Nemeroff. 2000. Neurobiology of posttraumatic stress disorder. Curr. Opin. Neurobiol. 10: 211-218.

68. ERICKSON, K., W. DREVETS \& J. SCHULKIN. 2003. Glucocorticoid regulation of diverse cognitive functions in normal and pathological emotional states. Neurosci. Biobehav. Rev. 27: 233-246. 
69. Оттошітz, W.E. et al. 2004. Neural and endocrine correlates of sadness in women: implications for neural network regulation of HPA activity. J. Neuropsychiatry Clin. Neurosci. 16: 446-455.

70. DREVETS, W.C. et al. 2002. Glucose metabolism in the amygdala in depression: relationship to diagnostic subtype and plasma cortisol levels. Pharmacol. Biochem. Behav. 71: 431-447.

71. BonNe, O. et al. 2003. Resting regional cerebral perfusion in recent posttraumatic stress disorder. Biol. Psychiatry 54: 1077-1086. 